\title{
Ethnicity modifies the association between diabetes mellitus and ischaemic heart disease in Chinese, Malays and Asian Indians living in Singapore
}

\author{
K. K. Yeo • B. C. Tai • D. Heng • J. M. J. Lee • S. Ma • \\ K. Hughes $\cdot$ S. K. Chew $\cdot$ K. S. Chia $\cdot$ E. S. Tai
}

Received: 30 May 2006 / Accepted: 14 August 2006 / Published online: 5 October 2006

(C) Springer-Verlag 2006

\begin{abstract}
Aims/hypothesis The aim of the study was to determine whether the risk of ischaemic heart disease (IHD) associated with diabetes mellitus differs between ethnic groups.

Methods Registry linkage was used to identify IHD events in 5707 Chinese, Malay and Asian Indian participants from three cross-sectional studies conducted in Singapore between the years 1984 and 1995. The study provided a median of 10.2 years of follow-up with 240 IHD events experienced. We assessed the interaction between diabetes mellitus and ethnicity in relation to the risk of IHD events using Cox proportional hazards regression.

Results Diabetes mellitus was more common in Asian Indians. Furthermore, diabetes mellitus was associated with a greater risk of IHD in Asian Indians. The hazard ratio when comparing diabetes mellitus with non-diabetes mellitus
\end{abstract}

Electronic supplementary material Supplementary material is available in the online version of this article at http://dx.doi.org/ $10.1007 / \mathrm{s} 00125-006-0469-\mathrm{z}$ and is accessible to authorised users.

K. K. Yeo $\cdot$ E. S. Tai $(\bowtie)$

Department of Endocrinology, Singapore General Hospital,

Block 6 level 6, Room B35, Outram Road,

Singapore 276692, Singapore

e-mail: eshyong@pacific.net.sg

B. C. Tai $\cdot$ J. M. J. Lee $\cdot$ K. S. Chia

Center for Molecular Epidemiology,

National University of Singapore,

Singapore, Singapore

D. Heng $\cdot \mathrm{S} . \mathrm{Ma} \cdot \mathrm{S}$. K. Chew

Ministry of Health,

Singapore, Singapore

K. Hughes

Department of Community, Occupational and Family Medicine,

National University of Singapore,

Singapore, Singapore was $6.41(95 \%$ CI 5.77-7.12) in Asian Indians and 3.07 (95\% CI 1.86-5.06) in Chinese ( $p=0.009$ for interaction). Differences in the levels of established IHD risk factors among diabetics from the three ethnic groups did not appear to explain the differences in IHD risk.

Conclusions/interpretation Asian Indians are more susceptible to the development of diabetes mellitus than Chinese and Malays. When Asian Indians do develop diabetes mellitus, the risk of IHD is higher than for Chinese and Malays. Consequently, the prevention of diabetes mellitus amongst this ethnic group is particularly important for the prevention of IHD in Asia, especially given the size of the population at risk. Elucidation of the reasons for these ethnic differences may help us understand the pathogenesis of IHD in those with diabetes mellitus.

Keywords Epidemiology · Cardiac complications . Clinical sciences
Abbreviations
HDL-C high-density-lipoprotein cholesterol
IHD ischaemic heart disease
LDL-C low-density-lipoprotein cholesterol

\section{Introduction}

Diabetes mellitus is a major risk factor for ischaemic heart disease (IHD). It has been found that, among those with diabetes mellitus, ethnicity affects the morbidity and mortality associated with this condition. It has been found that black people in both the USA [1, 2] and the UK [3] are less likely to develop IHD than their white counterparts.

The elucidation of these ethnic differences may be particularly relevant in Asia since this is the region where 
diabetes mellitus is likely to see the largest increase in prevalence over the next 5-10 years [4]. It is also the region where the burden of IHD is likely to increase the most over the next several decades [5, 6]. Unfortunately, the data among ethnic groups resident in Asia are limited. In the USA it has been reported that Asians (comprising primarily Chinese and Filipinos) with diabetes mellitus have a lower risk of IHD compared with their white counterparts $[2,7]$. In the UK, Asian Indians with diabetes mellitus have been reported to have a higher rate of IHD [8] than whites with diabetes mellitus in one study and similar rates in another [9].

It is also unclear why these ethnic differences in morbidity and mortality among individuals with diabetes mellitus exist. They could result from a difference in the baseline risk of IHD in the various ethnic groups which persist even in the presence of diabetes mellitus. Alternatively, ethnicity could modify the risk of IHD associated with diabetes mellitus. In other words, diabetes mellitus could have a greater effect on IHD risk in some ethnic groups than in others. Because most of the studies examining this issue have included only individuals with diabetes mellitus, without normal individuals for comparison, no conclusions can be drawn as to which of these mechanisms is operating in these populations. The aim of this study was to determine whether or not ethnicity modifies the risk of IHD associated with diabetes mellitus in Chinese, Malays and Asian Indians living in Singapore.

\section{Subjects and methods}

For this study we used data from the Singapore Cardiovascular Cohort Study. This study has been described previously $[10,11]$. The cohort was composed of participants from three previous cross-sectional surveys: the Thyroid and Heart Study 1982-1984 [12], the National Health Survey 1992 [13] and the National University of Singapore Heart Study 1993-1995 [14]. Details of the methods used in these three surveys have been described elsewhere [12-14]. The characteristics of the subjects from each of the original study populations are shown in the Electronic supplementary material, Table 1. Informed consent was obtained for all participants and the Study was approved by the Institutional Review Board of the National University of Singapore.

All subjects were examined in the morning following a 10-h overnight fast. Plasma glucose and serum lipid concentrations (total cholesterol, triacylglycerol and highdensity-lipoprotein cholesterol [HDL-C]) were measured. Low-density-lipoprotein cholesterol (LDL-C) was calculated using the Friedewald formula. Ninety-eight subjects (1.7\%) had a fasting triacylglycerol concentration above $4.5 \mathrm{mmol} / \mathrm{l}$ and LDL-C was therefore not calculated in these individuals. Diabetes mellitus was defined as a known history of diabetes mellitus on medication or a fasting plasma glucose concentration $\geq 7.0 \mathrm{mmol} / \mathrm{l}$. At least two readings of blood pressure were taken from respondents who had rested adequately before measurement, using a standard mercury sphygmomanometer. If the two readings differed by more than diastolic $15 \mathrm{mmHg}$ or systolic $25 \mathrm{mmHg}$, a third reading was performed. The mean values of the closest two readings were calculated. Hypertension was defined as having a history of hypertension on medication, or systolic blood pressure $\geq 140 \mathrm{mmHg}$ or diastolic blood pressure $\geq 90 \mathrm{mmHg}$. The BMI was calculated by dividing weight in $\mathrm{kg}$ by the square of height in $\mathrm{m}$. A smoker was defined as anyone who gave a history of smoking. Ethnicity was self-reported at study entry and was classified into Chinese, Malay, Asian Indian and Others.

Outcomes were obtained by linking individual records (using unique national registry identity card numbers) to three national registries. These were: (1) the Registry of Births and Deaths; (2) Hospital inpatient discharge databases; and (3) the Singapore Myocardial Infarct Registry, a populationbased registry with comprehensive coverage of acute myocardial infarction occurring in Singapore. All outcome measures were in coded form using the ninth revision of the International Classification of Diseases (ICD-9). An IHD event was defined as the occurrence of acute myocardial infarction or IHD (ICD-9 410-414) recorded in the registries mentioned above. In the time-to-IHD analysis, the time to IHD was defined as the difference between the date of first IHD and the date of entry into the respective studies. Subjects without IHD were censored at 31 December 2002 or the date of non-IHD death, whichever occurred first.

\section{Statistical analysis}

Statistical analyses were performed using STATA version 8.2 (StataCorp, College Station, Texas, USA, 2003). Continuous variables were compared using ANOVA (except for fasting triacylglycerol, which was compared using the Kruskal-Wallis test). Proportions were compared using the $\chi^{2}$ test. Kaplan-Meier survival curves were plotted to visually assess the relationship between time to IHD and prognostic factors. Comparisons of survival between groups were carried out using the log-rank test. Additional time-to-event analyses were conducted using Cox proportional hazards regression, with assumptions in regard to proportionality and linearity carefully checked. Age, when entered on its own, violated these assumptions and the square of age was therefore included in the model to overcome them. Hazard ratios and $95 \%$ CIs for IHD were estimated for risk factors of interest. The homogeneity of effect between ethnic groups was evaluated by including the interaction term involving diabetes mellitus and ethnic group in the model. The significance of this interaction 
Table 1 Levels of established risk factors for ischaemic heart disease according to diabetes status and ethnicity

\begin{tabular}{|c|c|c|c|c|c|c|c|c|}
\hline & \multicolumn{3}{|c|}{ Diabetes mellitus present } & \multirow{2}{*}{$\begin{array}{l}p \\
\text { value }\end{array}$} & \multicolumn{3}{|c|}{ Diabetes mellitus absent } & \multirow{2}{*}{$\begin{array}{l}p \\
\text { value }\end{array}$} \\
\hline & $\begin{array}{l}\text { Chinese } \\
(n=218)\end{array}$ & $\begin{array}{l}\text { Malay } \\
(n=100)\end{array}$ & $\begin{array}{l}\text { Indian } \\
(n=127)\end{array}$ & & $\begin{array}{l}\text { Chinese } \\
(n=3390)\end{array}$ & $\begin{array}{l}\text { Malay } \\
(n=1005)\end{array}$ & $\begin{array}{l}\text { Indian } \\
(n=867)\end{array}$ & \\
\hline No. of events & 18 & 15 & 30 & & 104 & 33 & 40 & \\
\hline $\begin{array}{l}\text { Median follow-up duration } \\
\text { (years) }\end{array}$ & $\begin{array}{l}10.24 \\
(7.20-20.23)\end{array}$ & $\begin{array}{l}10.23 \\
(7.03-19.94)\end{array}$ & $\begin{array}{l}10.20 \\
(7.06-19.52)\end{array}$ & & $\begin{array}{l}10.26 \\
(5.04-20.81)\end{array}$ & $\begin{array}{l}10.23 \\
(7.03-20.27)\end{array}$ & $\begin{array}{l}10.22 \\
(7.03-20.27)\end{array}$ & \\
\hline Age (years) & $52.3(11.7)$ & $51.3(11.5)$ & $50.7(11.4)$ & 0.451 & $37.4(12.6)$ & $36.5(13.1)$ & $37.6(12.7)$ & 0.100 \\
\hline Gender: $n$ ( $\%$ of males $)$ & $117(53.7)$ & $51(51.0)$ & $69(54.3)$ & 0.870 & $1,646(48.6)$ & $495(49.3)$ & $418(48.2)$ & 0.894 \\
\hline Smoker: $n(\%)$ & $76(34.9)$ & $39(39.0)$ & $40(31.5)$ & 0.811 & $953(28.1)$ & $370(36.8)$ & $206(23.8)$ & $<0.001$ \\
\hline \multicolumn{9}{|l|}{$\begin{array}{l}\text { Alcohol consumption: } \\
n(\%)\end{array}$} \\
\hline At least once per month & $21(9.6)$ & $2(2.0)$ & $17(13.4)$ & $<0.001$ & $384(11.3)$ & $15(1.5)$ & 99 (11.4) & $<0.001$ \\
\hline Occasionally & $46(21.1)$ & $5(5.0)$ & $19(15.0)$ & & $1,048(30.9)$ & $53(5.3)$ & $148(17.1)$ & \\
\hline Never & $151(69.3)$ & $93(93.0)$ & $91(71.7)$ & & $1,957(57.8)$ & $937(93.2)$ & $620(71.5)$ & \\
\hline BMI $\left(\mathrm{kg} / \mathrm{m}^{2}\right)$ & $25.3(3.72)$ & $27.6(4.84)$ & $26.2(4.60)$ & $<0.001$ & $22.3(3.67)$ & $24.1(4.62)$ & $24.2(4.67)$ & $<0.001$ \\
\hline Total cholesterol (mmol/l) & $6.13(1.23)$ & $6.41(1.39)$ & $5.98(1.22)$ & 0.039 & $5.42(1.15)$ & $5.60(1.12)$ & $5.42(1.08)$ & $<0.001$ \\
\hline Triacylglycerol (mmol/l) & $\begin{array}{l}1.81 \\
(0.61-12.75)\end{array}$ & $\begin{array}{l}1.82 \\
(0.63-10.32)\end{array}$ & $\begin{array}{l}1.72 \\
(0.44-9.10)\end{array}$ & 0.645 & $\begin{array}{l}1.03 \\
(0.39-5.09)\end{array}$ & $\begin{array}{l}1.13 \\
(0.43-4.11)\end{array}$ & $\begin{array}{l}1.14 \\
(0.40-4.30)\end{array}$ & $<0.001$ \\
\hline HDL-C (mmol/l) & $1.05(0.34)$ & $1.01(0.31)$ & $0.96(0.34)$ & 0.061 & $1.18(0.38)$ & $1.10(0.36)$ & $1.00(0.32)$ & $<0.001$ \\
\hline LDL-C (mmol/l) & $4.14(1.23)$ & $4.37(1.25)$ & $4.10(1.06)$ & 0.201 & $3.65(1.10)$ & $3.89(1.06)$ & $3.81(1.01)$ & $<0.001$ \\
\hline $\begin{array}{l}\text { Systolic blood pressure } \\
(\mathrm{mmHg})\end{array}$ & $118.0(18.1)$ & $119.7(19.2)$ & $117.4(17.6)$ & 0.012 & $134.8(23.3)$ & $140.0(27.4)$ & $132.0(20.7)$ & 0.040 \\
\hline $\begin{array}{l}\text { Diastolic blood pressure } \\
(\mathrm{mmHg})\end{array}$ & $70.2(11.9)$ & $71.7(12.4)$ & $70.2(12.4)$ & 0.002 & $79.6(11.2)$ & $81.1(12.4)$ & $77.3(11.9)$ & 0.040 \\
\hline Hypertension (\%) & $107(49.1)$ & $52(52.0)$ & $55(43.3)$ & 0.394 & $493(14.5)$ & $177(17.6)$ & $125(14.4)$ & 0.048 \\
\hline
\end{tabular}

All values for continuous variables are shown as mean (SD) except for follow-up duration and triacylglycerol, which are shown as median (range)

term was assessed using the log-likelihood ratio statistic. This was obtained by taking the difference between the values of $-2 \log$-likelihood for the model including the interaction term, and another model omitting it. The loglikelihood statistic has an asymptotic $\chi^{2}$ distribution, the number of degrees of freedom being equal to the difference between the number of parameters being fitted under the two models. The log hazard ratio associated with diabetes mellitus in each ethnic group was estimated by taking the additive effect of log hazard ratios associated with diabetes mellitus and the interaction term involving the specific ethnic group. The population-attributable risk was estimated using the formula:

$\lambda=\frac{\theta_{\varepsilon}(\phi-1)}{1+\theta_{\varepsilon}(\phi-1)}$

where $\lambda$ is the population-attributable risk, $\theta_{\varepsilon}$ is the proportion of individuals with diabetes mellitus and $\phi$ is the relative risk.

\section{Results}

There were 5920 patients in the cohort. Of these, 117 had pre-existing IHD and 17 had pre-existing cerebrovascular accidents. These subjects, together with another 36 with missing information on these fields ( 33 with missing fasting plasma glucose measurements, and ten who were of 'Other' ethnic origin), were excluded from the analysis. The median follow-up duration for the remaining 5707 patients analysed was 10.2 (range $0.04-20.8$ ) years. A total of 240 (Chinese 122, 3\%; Malay 48, 4\%; Asian Indian 70, 7\%) IHD events were reported.

The overall prevalence of diabetes mellitus was $6.0 \%$ in Chinese, $9.0 \%$ in Malays and $12.8 \%$ in Asian Indians. The characteristics of those with and without diabetes mellitus from each of the three ethnic groups are shown in Table 1. Among those without diabetes mellitus, Asian Indians had the lowest serum HDL-C concentration, followed by Malays and Chinese. This difference persisted even in those with diabetes mellitus and reached borderline statistical significance $(p=0.061)$. In contrast, the higher LDL-C and triacylglycerol concentrations observed in Asian Indians without diabetes mellitus was not evident in those with diabetes mellitus. In fact, Asian Indians appeared to exhibit lower levels of these risk factors. Asian Indians were also less likely to have hypertension and had lower blood pressure than their Malay and Chinese counterparts. Malays were also more likely to have a history of cigarette smoking and less likely to consume alcohol. 
We also compared the levels of various risk factors related to treatment in Chinese, Malays and Asian Indians with diabetes mellitus (Table 2). Unfortunately, the only data available with regard to treatment were whether or not patients were being followed up by their doctors for either diabetes mellitus or hypertension. No data were available on the specific drugs used in each ethnic group. Our data showed that Asian Indians with diabetes mellitus were more likely to be receiving treatment and follow-up by a physician for their condition. In addition, $\mathrm{HbA}_{1 \mathrm{c}}$ values were available for a small subset of subjects with known diabetes mellitus who participated in the 1992 National Health survey. These data showed that the level of glycaemic control in Asian Indians was as good as, if not better than, that in other ethnic groups, but this difference did not reach statistical significance. Among those with diabetes mellitus and hypertension, a similar proportion were receiving treatment for hypertension in all three ethnic groups and, among those receiving treatment, blood pressure was better controlled in Asian Indians than in the other two ethnic groups.

Figure 1 shows the Kaplan-Meier survival curves for those with and without diabetes mellitus in the three ethnic groups. In those with and without diabetes mellitus, Asian Indians had the poorest event-free survival rates, followed by Malays and Chinese, These differences were more pronounced in those with diabetes mellitus than in those without.

Table 3 shows the hazards ratios associated with the various risk factors for IHD. There is evidence of interaction between diabetes mellitus and ethnicity $\left(\chi^{2}=9.52, p=0.009\right)$. The additional risk of IHD in those with diabetes mellitus compared with those without diabetes mellitus was greater in Asian Indians (hazard ratio $6.41,95 \%$ CI 5.77-7.12) than in Chinese (hazard ratio $3.07,95 \%$ CI $1.86-5.06$ ). This difference persisted even after adjusting for established risk factors for IHD. Several different, additional models were considered (data not shown). In the first, in addition to the variables described in Table 3, we also considered a model that included variables encoding treatment for diabetes mellitus and treatment for hypertension. Both these variables were associated with reduced risk of IHD disease but did not alter the interaction between diabetes mellitus and ethnicity. We also considered a model that included these two treatment variables and the interactions terms diabetes mellitus $\times$ treatment for diabetes mellitus and hypertension $\times$ treatment for hypertension in order to consider the possibility that treatment of risk factors does not entirely eliminate adverse prospective effects. We could not assess the interaction between the presence of diabetes mellitus and treatment for diabetes mellitus because the terms were colinear. Consequently, these could only be included as main effects in the model. However, inclusion of variables for treatment or hypertension with blood pressure (either as a dichotomous variable [hypertension: yes vs no] or as a continuous variable [systolic blood pressure]) was possible and this interaction term was of borderline statistical significance. However, there was no impact on the interaction between diabetes mellitus and ethnicity.

\section{Discussion}

In this study we have shown that ethnic differences in IHD rates amongst those with diabetes mellitus result from a difference in the baseline risk of IHD between ethnic groups as well as a difference in the risk of IHD associated with diabetes mellitus between ethnic groups. This is the first time that ethnicity has been shown to modify the risk of IHD associated with diabetes mellitus. The ethnic group most affected in our population was the Asian Indians.

Asian Indians, even among non-diabetics, had a higher risk of IHD. This finding is in accordance with previous findings [11, 15-21]. As in previous studies, adjustment for other traditional risk factors did not entirely account for the increased risk of IHD in this ethnic group [11, 19]. Diabetes mellitus was also more prevalent in Asian Indians than other ethnic groups, a finding which is in line with the findings of several previous studies [12-14, 18, 22]. We and others have shown that Asian Indians, compared with those in other ethnic groups, are also more insulin-resistant $[13,14,23,24]$ and exhibit more risk factors associated

Table 2 Control of cardiovascular risk factors amongst subjects with diabetes mellitus according to ethnic group

\begin{tabular}{|c|c|c|c|c|}
\hline & $\begin{array}{l}\text { Chinese } \\
(n=218)\end{array}$ & $\begin{array}{l}\text { Malay } \\
(n=100)\end{array}$ & $\begin{array}{l}\text { Asian Indian } \\
(n=127)\end{array}$ & $p$ value \\
\hline Number $(\%)$ of diabetics receiving treatment for diabetes mellitus & $103(47)$ & $53(53)$ & $75(59)$ & 0.103 \\
\hline $\mathrm{HbA}_{1 \mathrm{c}}(\%)$ in diabetics receiving treatment for diabetes mellitus ${ }^{\mathrm{a}}$ & $8.7(8.3-9.2)$ & $8.6(7.4-9.8)$ & $8.3(7.3-9.4)$ & 0.779 \\
\hline Number (\%) of hypertensive subjects receiving treatment for hypertension & $76(71)$ & $34(65)$ & $37(67)$ & 0.745 \\
\hline Systolic blood pressure in subjects receiving treatment for hypertension $(\mathrm{mmHg})$ & $154.1(23.7)$ & $165.2(27.7)$ & $149.4(22.0)$ & 0.020 \\
\hline Diastolic blood pressure in subjects receiving treatment for hypertension $(\mathrm{mmHg})$ & $87.4(10.5)$ & $91.5(9.9)$ & $87.4(11.7)$ & 0.146 \\
\hline
\end{tabular}

${ }^{\mathrm{a}} \mathrm{HbA}_{1 \mathrm{c}}$ was available only in those with known diabetes mellitus in the 1992 National Health Survey ( $n=89$ for Chinese, $n=21$ for Malays and $n=20$ for Asian Indians) 


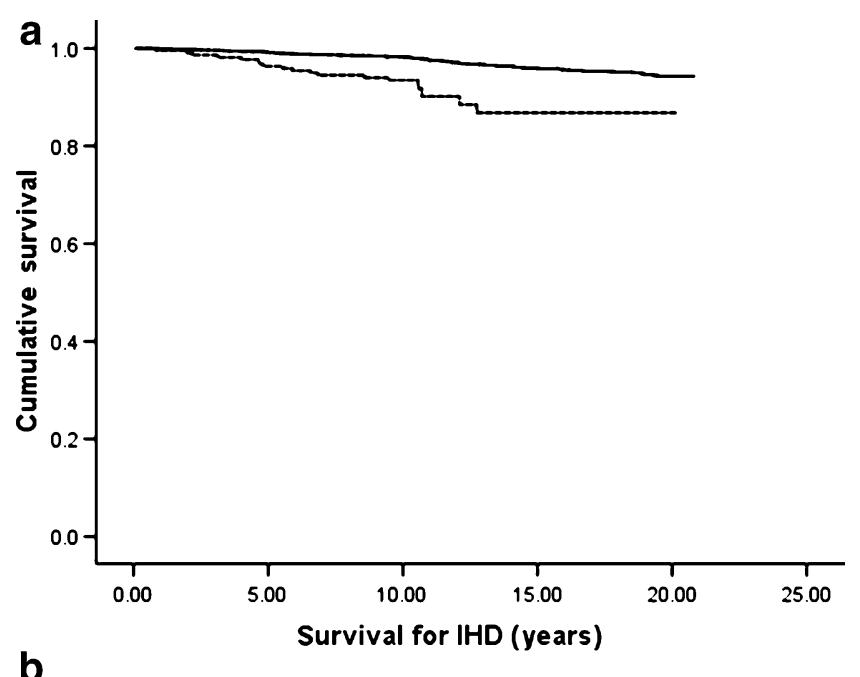

and environmental factors resulting in greater susceptibility to these conditions when exposed to a modern, urban environment such as that in Singapore [27].

To make matters worse, we have now observed that, over and above the increased baseline risk of IHD and the higher prevalence of diabetes mellitus in Asian Indians, diabetes mellitus had a larger effect on the risk of IHD in Asian Indians than in Chinese and Malays. As a consequence of the higher prevalence of diabetes mellitus and the greater effect of diabetes mellitus on the risk of IHD, the population-attributable risk of IHD related to diabetes mellitus was $40.9 \%$ in Asian Indians compared with $27.9 \%$ in Malays and $11.0 \%$ in Chinese.

The greater risk of IHD among Asian Indians with diabetes mellitus is unexplained at this time. One possibility is that Asian Indians may develop diabetes mellitus at an earlier age [28], resulting in more prolonged exposure to the deleterious effects of diabetes mellitus in Asian Indians. In line with this hypothesis, it has been shown that the relative risk of cardiovascular disease was greater in those who developed diabetes mellitus at a younger age than in those who developed it when they were older [29]. Unfortunately, we have data on the age of onset of diabetes only in a subgroup of this study population and, although the age of onset of diabetes was earlier in Asian Indians, the differences between ethnic groups were not statistically significant (data not shown). The testing of this hypothesis is complicated by the difficulties in determining the precise time of onset of diabetes mellitus because it often remains asymptomatic for many years before diagnosis [30].

Alternatively, it is possible that diabetes mellitus and its associated cardiovascular risk factors may be less well controlled in Asian Indians than in Chinese and Malays. In the USA it has been reported that diabetes mellitus is less well controlled in ethnic minorities [7, 31-33] than in whites. We attempted to assess this in our study to see whether this could explain the interaction between diabetes mellitus and ethnicity that was observed.

In our study it is true that Asian Indians with diabetes mellitus had slightly lower HDL-C than Chinese with diabetes mellitus. However, in relation to other established risk factors for IHD, they appeared to have a less atherogenic risk profile. They had lower LDL-C concentration, which in the UK Prospective Diabetes Study was the most important predictor of IHD events in a diabetic population [34]. They were also less likely to smoke, experienced less hypertension and were more likely to ingest alcohol. In contrast, the Malays, who had higher LDL-C and were more likely to be hypertensive than the Chinese, did not experience the same increase in risk of IHD associated with diabetes mellitus as the Asian Indians.

We also attempted to ascertain the effects of treatment on the risk of IHD in those with diabetes mellitus, and whether 
Table 3 Crude and adjusted (for all other risk factors in table) hazard ratio estimates of selected risk factors for IHD

\begin{tabular}{llc}
\hline & Crude hazard ratio (95\% CI) & Adjusted hazard ratio (95\% CI) \\
\hline Age (years) & $1.08(1.07-1.09)$ & $1.25(1.15-1.37)$ \\
Square of age (years) & - & $0.998(0.997-0.999)$ \\
Sex (male versus female) & $1.82(1.40-2.37)$ & $1.82(1.29-2.58)$ \\
Alcohol consumption & & $0.46(0.27-0.79)$ \\
At least once per month & $0.67(0.40-1.09)$ & $0.65(0.44-0.95)$ \\
Occasionally & $0.59(0.42-0.82)$ & $1.39(1.00-1.92)$ \\
Smoking history (yes vs no) & $1.78(1.38-2.30)$ & $1.05(1.02-1.09)$ \\
BMI (kg/m $\left.{ }^{2}\right)$ & $1.12(1.09-1.14)$ & $0.60(0.37-0.95)$ \\
HDL-C (mmol/l) & $0.35(0.24-0.51)$ & $1.30(1.16-1.45)$ \\
LDL-C (mmol/l) & $1.55(1.42-1.70)$ & $2.17(1.60-2.92)$ \\
Hypertension (yes vs no) & $7.00(5.42-9.04)$ & $0.74(0.42-1.30)$ \\
Diabetes mellitus (yes vs no) & & $1.40(0.73-2.69)$ \\
Chinese & $3.07(1.86-5.06)$ & $2.29(1.40-3.73)$ \\
Malay & $5.30(3.56-7.90)$ & \\
Indian & $6.41(5.77-7.12)$ & \\
\hline
\end{tabular}

Analysis was carried out using Cox proportional hazards regression

The log hazard ratio associated with diabetes in each ethnic group was estimated by taking the additive effect of log hazard ratios associated with diabetes mellitus and the interaction term involving the specific ethnic group

Homogeneity of effect between ethnic groups was tested using the log likelihood ratio statistic and was significant $(p=0.009)$

such treatment might alter the risk of IHD associated with diabetes mellitus in each ethnic group. Unfortunately, our ability to make this assessment in this study population was limited. Although we were able to identify individuals who were receiving treatment for either diabetes mellitus or hypertension by a physician, data on the specific drugs used, including any that may have been used to treat dyslipidaemia were not available. Nevertheless, based on these data, it appeared that a greater proportion of Asian Indians with diabetes mellitus were receiving treatment and that the level of glycaemic control in this group was similar or better than that in Chinese or Malays. Among those with diabetes mellitus, the proportion of hypertensive subjects receiving treatment was also similar between ethnic groups. Adjusting for treatment for diabetes mellitus and hypertension did not alter the interaction between diabetes mellitus and ethnicity that we observed. Two other studies in Singapore have explored ethnic differences in diabetes mellitus-related parameters $[35,36]$. In terms of glycaemic control, the data are inconclusive. One study showed that Malays had the highest $\mathrm{HbA}_{1 \mathrm{c}}$ and that Chinese and Asian Indians had similar levels [36], whereas an earlier study showed that Asian Indians with diabetes mellitus had higher $\mathrm{HbA}_{1} \mathrm{c}$ than Chinese and Malays [35]. The study by Hong et al. [35] is particularly interesting because it showed that Asian Indians exhibited a higher prevalence of IHD but a lower prevalence of retinopathy and albuminuria, each of which is associated with poor control of diabetes mellitus and its associated metabolic derangements. Given these previous findings, it is a little difficult to suggest that poor control of diabetes mellitus accounts for the higher rates of IHD in Asian Indians but does not cause high rates of other complications, which are also associated with poor control.

It is also possible that the interaction observed relates not to diabetes mellitus per se but to an interaction between ethnicity and one of the other CVD risk factors associated with diabetes mellitus, such as hypertension or dyslipidaemia. However, this study was designed specifically to test the hypothesis that diabetes mellitus and ethnicity interact in relation to the risk of IHD and, given the limited power of this study to examine multiple interactions, we have not examined any additional interactions.

Our study has several strengths. The study population represents several random samples of the Singapore population. Singapore is a small island nation (land area $647 \mathrm{~km}^{2}$ or 250 square miles) which is completely urbanised, with three ethnic groups living in close proximity, each of which experienced urbanisation at the same time. The standard of living is relatively high and comparable between ethnic groups, the median monthly household income being US\$1504 for Malays, US\$1882 for Indians and US\$2138 for Chinese [17]. These features of the population enable us to avoid some of the problems associated with the effects of geography [1] or socioeconomic status and their impact on IHD risk that are seen in other studies.

Nevertheless, there are limitations to this study that need to be borne in mind when considering the implications of our findings. Firstly, the ascertainment of IHD events in this study was carried out using data from several registries, which could be unreliable. We are currently unable to provide direct evidence about the reliability of the registry data. However, we can say that data from the myocardial 
infarction registry were derived using the WHO MONICA protocol. The methodology is therefore well established. Furthermore, submissions to hospital in-patient discharge databases are a regulatory requirement for all hospitals in Singapore and most, if not all, hospital admissions are represented. The discharge diagnoses are completed by doctors directly involved in the management of the patients admitted to the hospital and we believe that they are reasonably accurate. Second, the high risk of IHD in Asian Indians has been known for many years, and an increased tendency to diagnose IHD amongst Asian Indians could certainly bias the findings towards a higher risk of IHD in this ethnic group. However, these findings have now been replicated in at least three different populations and we have reason to believe that the ethnic differences in the baseline risk of IHD are real. Furthermore, the interaction between ethnicity and diabetes mellitus is a novel finding of this study and has not been reported in any population to date. Therefore, we have no reason to believe that the interaction observed is related to such misclassification, which is not thought to be particularly prominent in Asian Indians with diabetes mellitus. Finally, the IHD risk factors examined in this study were measured only at a single time point. This could give rise to significant misclassification. However, we believe that this misclassification is likely to be non-differential as the measurements were made prospectively and therefore are likely to bias the associations towards the null hypothesis (which in this instance is the hypothesis of no interaction). Consequently, we feel that any such misclassification is unlikely to alter the conclusions based on the observation that diabetes mellitus does interact with ethnicity in relation to the risk of IHD.

In conclusion, our study shows that Asian Indians are particularly prone to the effects of diabetes mellitus on IHD risk for several reasons. First, they have a higher baseline risk of IHD. Second, diabetes mellitus is more common among Asian Indians. Finally, the effect of diabetes mellitus on the risk of IHD in this ethnic group is greater than in other ethnic groups. Several studies have shown that the prevention of diabetes mellitus is not only possible [37-40] but is also cost-effective [41]. Given the large population at risk in Asia and the large population-attributable risk of IHD associated with diabetes mellitus in this ethnic group, the development of programmes to prevent diabetes mellitus amongst Asian Indians may be particularly pertinent to any successful strategies to reduce the morbidity and mortality from cardiovascular disease in Asia. In addressing these ethnic differences, it is important to realise that ethnicity (unlike race) is a construct that encompasses both genetic and cultural (e.g. language, religion, diet) differences [42, 43]. We have to be careful not to assume that these observations are due solely to underlying genetic differences. Clearly, ethnic differences in other factors, such as the rates of other complications that may increase risk of IHD (e.g. diabetic nephropathy) and types of treatment used (especially lipid-lowering therapy), are inadequately explored in this study and should form the basis of future research in this field. The elucidation of the reasons for these ethnic differences may help us understand the pathogenesis of IHD in those with diabetes mellitus and identify novel treatment and prevention strategies.

\section{References}

1. Bertoni AG, Kirk JK, Case LD et al (2005) The effects of race and region on cardiovascular morbidity among elderly Americans with diabetes. Diabetes Care 28:2620-2625

2. Karter AJ, Ferrara A, Liu JY, Moffet HH, Ackerson LM, Selby JV (2002) Ethnic disparities in diabetic complications in an insured population. JAMA 287:2519-2527

3. Chaturvedi N, Jarrett J, Morrish N, Keen H, Fuller JH (1996) Differences in mortality and morbidity in African Caribbean and European people with non-insulin dependent diabetes mellitus: results of 20 year follow up of a London cohort of a multinational study. Br Med J 313:848-852

4. Zimmet P, Alberti KG, Shaw J (2001) Global and societal implications of the diabetes epidemic. Nature 414:782-787

5. Murray CJ, Lopez AD (1997) Alternative projections of mortality and disability by cause 1990-2020: Global Burden of Disease Study. Lancet 349:1498-1504

6. Reddy KS, Yusuf S (1998) Emerging epidemic of cardiovascular disease in developing countries. Circulation 97:596-601

7. Lanting LC, Joung IM, Mackenbach JP, Lamberts SW, Bootsma $\mathrm{AH}$ (2005) Ethnic differences in mortality, end-stage complications, and quality of care among diabetic patients: a review. Diabetes Care 28:2280-2288

8. Mather HM, Chaturvedi N, Fuller JH (1998) Mortality and morbidity from diabetes in South Asians and Europeans: 11-year follow-up of the Southall Diabetes Survey, London, UK. Diabet Med 15:53-59

9. Samanta A, Burden AC, Jagger C (1991) A comparison of the clinical features and vascular complications of diabetes between migrant Asians and Caucasians in Leicester, U.K. Diabetes Res Clin Pract 14:205-213

10. Heng diabetes mellitus, Lee J, Chew SK, Tan BY, Hughes K, Chia KS (2000) Incidence of ischaemic heart disease and stroke in Chinese, Malays and Indians in Singapore: Singapore Cardiovascular Cohort Study. Ann Acad Med Singapore 29:231-236

11. Lee J, Heng D, Chia KS, Chew SK, Tan BY, Hughes K (2001) Risk factors and incident coronary heart disease in Chinese, Malay and Asian Indian males: the Singapore Cardiovascular Cohort Study. Int J Epidemiol 30:938-983

12. Hughes K, Yeo PP, Lun KC et al (1990) Cardiovascular diseases in Chinese, Malays, and Indians in Singapore. II. Differences in risk factor levels. J Epidemiol Community Health 44:29-35

13. Tan CE, Emmanuel SC, Tan BY, Jacob E (1999) Prevalence of diabetes and ethnic differences in cardiovascular risk factors. The 1992 Singapore National Health Survey. Diabetes Care 22:241-247

14. Hughes K, Aw TC, Kuperan P, Choo M (1997) Central obesity, insulin resistance, syndrome $\mathrm{X}$, lipoprotein(a), and cardiovascular risk in Indians, Malays, and Chinese in Singapore. J Epidemiol Community Health 51:394-399

15. McKeigue PM, Miller GJ, Marmot MG (1989) Coronary heart disease in south Asians overseas: a review. J Clin Epidemiol 42:597-609 
16. Hughes K, Lun KC, Yeo PP (1990) Cardiovascular diseases in Chinese, Malays, and Indians in Singapore. I. Differences in mortality. J Epidemiol Community Health 44:24-88

17. Mak KH, Chia KS, Kark JD et al (2003) Ethnic differences in acute myocardial infarction in Singapore. Eur Heart J 24:151-160

18. Anand SS, Yusuf S, Vuksan V et al (2000) Differences in risk factors, atherosclerosis and cardiovascular disease between ethnic groups in Canada: the study of health assessment and risk in ethnic groups (SHARE). Indian Heart J 52(Suppl 7): S35-S43

19. McKeigue PM, Ferrie JE, Pierpoint T, Marmot MG (1993) Association of early-onset coronary heart disease in South Asian men with glucose intolerance and hyperinsulinemia. Circulation $87: 152-161$

20. Tillin T, Forouhi N, Johnston DG, McKeigue PM, Chaturvedi N, Godsland IF (2005) Metabolic syndrome and coronary heart disease in South Asians, African-Caribbeans and white Europeans: a UK population-based cross-sectional study. Diabetologia 48:649-656

21. Sheth T, Nair C, Nargundkar M, Anand S, Yusuf S (1999) Cardiovascular and cancer mortality among Canadians of European, south Asian and Chinese origin from 1979 to 1993: an analysis of 1.2 million deaths. CMAJ 161:132-138

22. McKeigue PM, Shah B, Marmot MG (1991) Relation of central obesity and insulin resistance with high diabetes prevalence and cardiovascular risk in South Asians. Lancet 337:382-386

23. Abate N, Chandalia M, Snell PG, Grundy SM (2004) Adipose tissue metabolites and insulin resistance in nondiabetic Asian Indian men. J Clin Endocrinol Metab 89:2750-2755

24. Chandalia M, Abate N, Garg A, Stray-Gundersen J, Grundy SM (1999) Relationship between generalized and upper body obesity to insulin resistance in Asian Indian men. J Clin Endocrinol Metab 84:2329-2335

25. Heng D, Ma S, Lee JJ et al (2006) Modification of the NCEP ATP III definitions of the metabolic syndrome for use in Asians identifies individuals at risk of ischemic heart disease. Atherosclerosis 186:367-373

26. Tan CE, Ma S, Wai D, Chew SK, Tai ES (2004) Can we apply the National Cholesterol Education Program Adult Treatment Panel definition of the metabolic syndrome to Asians? Diabetes Care 27:1182-1186

27. Tai ES, Tan CE (2004) Genes, diet and serum lipid concentrations: lessons from ethnically diverse populations and their relevance to coronary heart disease in Asia. Curr Opin Lipidol 15:5-12

28. Tai ES, Lim SC, Chew SK, Tan BY, Tan CE (2000) Homeostasis model assessment in a population with mixed ethnicity: the 1992 Singapore National Health Survey. Diabetes Res Clin Pract 49:159-168
29. Woodward M, Zhang X, Barzi F et al (2003) The effects of diabetes on the risks of major cardiovascular diseases and death in the Asia-Pacific region. Diabetes Care 26:360-366

30. Harris MI, Klein R, Welborn TA, Knuiman MW (1992) Onset of NIDdiabetes mellitus occurs at least 4-7 yr before clinical diagnosis. Diabetes Care 15:815-889

31. Adams AS, Zhang F, Mah C et al (2005) Race differences in longterm diabetes management in an HMO. Diabetes Care 28:2844-2849

32. Bonds DE, Zaccaro DJ, Karter AJ, Selby JV, Saad M, Goff DC Jr (2003) Ethnic and racial differences in diabetes care: the Insulin Resistance Atherosclerosis Study. Diabetes Care 26:1040-1406

33. Harris MI (2001) Racial and ethnic differences in health care access and health outcomes for adults with type 2 diabetes. Diabetes Care 24:159-454

34. Turner RC, Millns H, Neil HA et al (1998) Risk factors for coronary artery disease in non-insulin dependent diabetes mellitus: United Kingdom Prospective Diabetes Study (UKPDS: 23). Br Med J 316:823-828

35. Hong CY, Chia KS, Hughes K, Ling SL (2004) Ethnic differences among Chinese, Malay and Indian patients with type 2 diabetes mellitus in Singapore. Singapore Med J 45:154-160

36. Ng TP, Goh LG, Tan Y et al (2005) Ethnic differences in glycaemic control in adult Type 2 diabetic patients in primary care: a 3-year follow-up study. Diabet Med 22:1598-1604

37. Chiasson JL, Josse RG, Gomis R, Hanefeld M, Karasik A, Laakso M (2002) Acarbose for prevention of type 2 diabetes mellitus: the STOP-NIDDM randomised trial. Lancet 359:2072-2077

38. Knowler WC, Barrett-Connor E, Fowler SE et al (2002) Reduction in the incidence of type 2 diabetes with lifestyle intervention or metformin. N Engl J Med 346:393-403

39. Pan XR, Li GW, Hu YH et al (1997) Effects of diet and exercise in preventing NIDdiabetes mellitus in people with impaired glucose tolerance. The Da Qing IGT and Diabetes Study. Diabetes Care 20:537-544

40. Tuomilehto J, Lindstrom J, Eriksson JG et al (2001) Prevention of type 2 diabetes mellitus by changes in lifestyle among subjects with impaired glucose tolerance. N Engl J Med 344: $1343-1350$

41. Hernan WH, Brandle M, Zhang P et al (2003) Costs associated with the primary prevention of type 2 diabetes mellitus in the diabetes prevention program. Diabetes Care 26:36-47

42. Anand SS (1999) Using ethnicity as a classification variable in health research: perpetuating the myth of biological determinism, serving socio-political agendas, or making valuable contributions to medical sciences? Ethn Health 4:241-244

43. Chaturvedi N (2001) Ethnicity as an epidemiological determinantcrudely racist or crucially important? Int J Epidemiol 30:925-927 